\title{
The altered gut virome community in rhesus monkeys is correlated with the gut bacterial microbiome and associated metabolites
}

Heng $\mathrm{Li}^{1,2+}$, Hongzhe $\mathrm{Li}^{1,2+}$, Jingjing Wang ${ }^{1,2}$, Lei Guo ${ }^{1,2}$, Haitao Fan ${ }^{1,2}$, Huiwen Zheng ${ }^{1,2}$, Zening Yang ${ }^{1,2}$, Xing Huang ${ }^{1,2}$, Manman Chu ${ }^{1,2}$, Fengmei Yang ${ }^{1}$, Zhanlong He${ }^{1}$, Nan $\mathrm{Li}^{1,2}$, Jinxi Yang ${ }^{1,2}$, Qiongwen $\mathrm{Wu}^{1,2}$, Haijing Shi ${ }^{1,2^{*}}$ and Longding Liu ${ }^{1,2^{*}}$

\begin{abstract}
Background: The gut microbiome is closely associated with the health of the host; although the interaction between the bacterial microbiome and the whole virome has rarely been studied, it is likely of medical importance. Examination of the interactions between the gut bacterial microbiome and virome of rhesus monkey would significantly contribute to revealing the gut microbiome composition.

Methods: Here, we conducted a metagenomic analysis of the gut microbiome of rhesus monkeys in a longitudinal cohort treated with an antibiotic cocktail, and we documented the interactions between the bacterial microbiome and virome. The depletion of viral populations was confirmed at the species level by real-time PCR. We also detected changes in the gut metabolome by GC-MS and LC-MS.
\end{abstract}

Results: A majority of bacteria were depleted after treatment with antibiotics, and the Shannon diversity index decreased from 2.95 to 0.22. Furthermore, the abundance-based coverage estimator (ACE) decreased from 104.47 to 33.84, and the abundance of eukaryotic viruses also changed substantially. In the annotation, 6 families of DNA viruses and 1 bacteriophage family were present in the normal monkeys but absent after gut bacterial microbiome depletion. Intriguingly, we discovered that changes in the gut bacterial microbiome composition may promote changes in the gut virome composition, and tryptophan, arginine, and quinone may play roles in the interaction between the bacterial microbiome and virome.

Conclusion: Our results indicated that the clearly altered composition of the virome was correlated with depletion in the bacterial community and that metabolites produced by bacteria possibly play important roles in the interaction.

Keywords: Gut viral community, Rhesus monkeys, Metagenomic analysis, Bacterial microbiome, Metabolite analysis, Correlation

\footnotetext{
* Correspondence: haijingshi@hotmail.com; longdingl@gmail.com

${ }^{\dagger}$ Heng Li and Hongzhe Li contributed equally to this work.

${ }^{1}$ Institute of Medical Biology, Chinese Academy of Medical Sciences \& Peking

Union Medical College, Kunming 650118, China

Full list of author information is available at the end of the article
}

(c) The Author(s). 2019 Open Access This article is distributed under the terms of the Creative Commons Attribution 4.0 International License (http://creativecommons.org/licenses/by/4.0/), which permits unrestricted use, distribution, and reproduction in any medium, provided you give appropriate credit to the original author(s) and the source, provide a link to the Creative Commons license, and indicate if changes were made. The Creative Commons Public Domain Dedication waiver (http://creativecommons.org/publicdomain/zero/1.0/) applies to the data made available in this article, unless otherwise stated. 


\section{Background}

The gut microbiome of an animal consists of bacteria, viruses, fungi and so on. This intricate ecosystem interacts with the adjacent epithelial layer, and the microbes perform metabolic functions, protect against pathogens, and condition the immune system, and through these basic functions, these microbes directly or indirectly affect most of the physiological functions of the host $[1,2]$. In recent years, variations in the bacterial community composition have been shown to correlate with infection outcome, inflammatory bowel disease [3], diabetes [4], obesity [5], and depression [6], and fecal microbiome transplantation has become an effective treatment for refractory Clostridium difficile infections and other diseases [7, 8]. The mechanisms of interaction between the gut bacterial microbiome and the host are very complex, and other components also play crucial roles in this process. In addition to bacteria, viruses are also abundant in the gut [9] and have been hypothesized to markedly alter the structure and function of the bacterial community [10-12]. Additionally, chronic viral infection can confer increased resistance against pathogenic challenges [13]. Gut virome alteration has been observed in inflammatory diseases such as inflammatory bowel disease and Crohn's disease [14]. The recent advent of highthroughput sequencing methods has made it possible to study these communities and their relationships with health and disease in detail [15].

Bacterial communities play an essential role in host health, but further research is still warranted to obtain an in-depth understanding of the mechanisms underlying this role. Transfer of whole virome communities between humans was documented in fecal microbiome transplantation [16], and the difference varied more widely between gut viromes than between gut bacterial microbiomes in humans [17]. However, the relationship between the bacterial microbiome and the virome has rarely been studied, despite its likely medical importance. Previous research has shown close relationships between single viral species and single bacterial species $[18,19]$, and single viral species could trigger shifts in the bacterial microbiome and the virome $[20,21]$. At the same time, enteric bacteria were seen to be required for efficient infection by [22, 23] or suppression of [24] viruses, and the richness of the gut bacterial microbiome had an obvious effect on bacteriophage composition [25]; moreover, the gut virome composition in humans was examined, and bacteriophage diversity was found to be inversely correlated with naturally occurring bacterial diversity in human infants during healthy development [26]. However, few studies have focused on how the whole virome, a diverse community consisting of eukaryotic RNA and DNA viruses and bacteriophages, interacts with the bacterial microbiome.

Rhesus monkeys are good mammalian research models that are closely related to humans, and the virome composition of these animals was seen to be affected by simian immunodeficiency virus infection [20]. We hypothesized that there is a close relationship between the whole gut virome and bacterial microbiome, and the bacterial microbiome could be depleted by treatment with an antibiotic cocktail in rhesus monkeys. We then examined the virome composition to detect the direct effects of the bacterial microbiota on the virome. We performed $16 \mathrm{~S}$ rRNA amplicon sequencing of the fecal bacteria and metagenomic analysis of fecal viromes from rhesus monkeys treated with an antibiotic cocktail. Our results suggest that a majority of bacteria were depleted after the monkeys were treated with antibiotics and that the composition of the whole virome changed drastically. Importantly, alteration of the virome along with shifts in the composition and function of the gut bacterial community and metabolites from gut bacteria may have played an important role in the interaction.

\section{Materials and methods Animals}

The rhesus monkey cohort described in this study was housed at the Institute of Medical Biology, Chinese Academy of Medical Sciences (IMBCAMS). An antibiotic cocktail containing ampicillin, streptomycin, kanamycin, metronidazole, and vancomycin was administered orally at a dose of $15 \mathrm{mg} /$ kg 3 times per day for 2 weeks. Three healthy one-year-old rhesus monkeys were treated with antibiotics, and fresh fecal samples were collected one day before treatment with antibiotics and 5, 8, and 9 days after treatment with antibiotics and stored at $-80^{\circ} \mathrm{C}$ for subsequent analysis. Fresh fecal samples from an additional three normal monkeys were collected after the monkeys were treated with antibiotics for 9 days. The bacterial community of each sample was detected by $16 \mathrm{~S}$ rRNA amplicon sequencing, and the virome communities in the samples collected before treatment with antibiotics and in those collected after treatment with antibiotics for 9 days were detected by deep sequencing. Because metabolome analysis requires 6 biological duplications, the metabolomes of samples collected before treatment with antibiotics and of those collected after treatment with antibiotics for 8 and 9 days were detected by GC-MS and LC-MS. In our analysis, samples collected before treatment with antibiotics were used as the control group, and samples collected after treatment with antibiotics were used as the experimental group.

\section{Bacterial 16S rRNA amplicon sequencing}

DNA was extracted from fecal samples, and PCR was performed with the barcode primers 338F/806R to obtain amplicons of hypervariable regions V3 and V4 for phylogenetic discrimination analysis [27]. Libraries were pooled by using a TruSeqTM DNA Sample Prep Kit and sequenced using an Illumina MiSeq sequencer. Sequences 
were assigned to closed-reference operational taxonomic units (OTUs) at a 97\% identity threshold using bacterial $16 \mathrm{~S}$ rRNA amplicon sequences from the Silva 128/16Sbacteria database. The OTU data were rarefied to the smallest effective sample sizes [28]; rarefaction is a homogenization method that is used to randomly draw OTUs to the same quantity based on a minimum value. The $\alpha$ diversity, which includes the abundance-based coverage estimator (ACE) and Shannon diversity index, was analyzed by Mothur (version v.1.30.1), and statistical significance was evaluated by Student's t-test.

\section{Kyoto encyclopedia of genes and genomes (KEGG) prediction analysis of the bacterial microbiome $[29,30]$} We performed a Kyoto Encyclopedia of Genes and Genomes (KEGG) prediction analysis of the bacterial microbiome using PICRUSt. PICRUSt contains the Cluster of Orthologous Groups of proteins (COG) and KEGG Ortholog (KO) information corresponding to Greengene ID numbers. For metagenome prediction, PICRUSt takes an input OTU table containing identifiers that match tips from the marker gene with corresponding abundances for each of the OTUs across one or more samples. First, PICRUSt normalizes the OTU table based on 16S rRNA amplicon copy number prediction so that the OTU abundances accurately reflect the true abundances of the underlying organisms. The metagenome is then predicted by looking up the precalculated genome content for each OTU, multiplying the normalized OTU abundance by each $\mathrm{KO}$ abundance in the genome and summing these $\mathrm{KO}$ abundances together per sample. The prediction yields a table of $\mathrm{KO}$ abundances for each metagenome sample in the OTU table.

\section{Analysis of similarities (ANOSIM)}

Analysis of similarities (ANOSIM) is a nonparametric test that shows whether the difference between groups is greater than that within groups. The analyses were performed in vegan or QIIME in R (version3.2.2) by using the Bray-Curtis algorithm [31].

\section{Virome DNA and RNA purification and sequencing}

Fecal samples were suspended in phosphate-buffered saline (PBS) and filtered through a filter with a pore size of $0.45 \mu \mathrm{m}$ (Millipore). The supernatant was enriched by a 30-kDa molecular mass filter (Ultra-15 $30 \mathrm{~K}$, Millipore). The concentrate was treated with DNase I (TaKaRa) at $37^{\circ} \mathrm{C}$ for $30 \mathrm{~min}$ to eliminate unencapsulated nucleic acids. Subsequently, total viral DNA was extracted from half of the concentrate using the QIAamp DNA Stool Kit (Qiagen), and at the same time, total viral RNA was extracted from the other half using the QIAamp Viral RNA Kit (Qiagen). The extracted RNA was synthesized into double strands using the NEBNext RNA First
Strand Synthesis Module (NEB) and the NEBNext mRNA Second Strand Synthesis Module (NEB). The DNA and double-stranded cDNA were amplified by whole-genome amplification (REPLI-g Mini Kit, Qiagen) and then fragmented into approximately 300-bp fragments by a Covaris M220 instrument. Then, the fragments were amplified into a PE library by the TruSeq DNA Sample Prep Kit and fixed to the chip by bridge PCR using the HiSeq 3000/4000 PE Cluster Kit. The constructs were sequenced on the Illumina HiSeq platform using HiSeq 3000/4000 SBS Kits.

For virome analysis, we first sheared the adaptor sequences with Seqprep and removed reads that were shorter than $50 \mathrm{bp}$ and those that contained $\mathrm{N}$ bases with Sickle to retain the paired-end reads and single-end reads. We compared these reads to the host (rhesus monkey) genome by BWA and removed the reads belonging to the host. Then, we compared all the clean reads with the U.S. National Center for Biotechnology Information (NCBI) Nucleotide database to identify the sequences that belonged to viruses and the sequences that did not belong to any known genome, such as those of bacteria, fungi or other known microorganisms. Then, contigs were built from these reads. The contigs and all reads that could not be mapped to any known genome in NCBI were compared with the virus protein database in the NCBI nonredundant RefSeq database (including sequences from SwissProt, PIR, PRF, and PDB and coding sequences (CDS) from GenBank and RefSeq) based on amino acid sequences using BLASTP (BLAST version 2.2.31+, e-value: 1e-5). These results constituted our virus database and were used to obtain the nonredundant gene catalog by CD-HIT. All the reads were compared to our virus database to analyze their richness. The spliced read alignments were predicted by MetaGene, compared to the EggNOG database and Virulence Factors database (VFDB) for COG analysis using BLASTP (BLAST version 2.2.31+, e-value: 1e-5) and annotated using VFDB.

\section{PCR validation}

The abundance results that were similar in 2 or more monkeys were selected, and real-time PCR was used to validate the changes in these viruses (SYBR Premix Ex Taq II, TaKaRa). As the template, we used the DNA and cDNA extracted from fecal samples. The samples that were not detected directly from the DNA or cDNA were subjected to multiple displacement amplification (MDA) (total nucleic acid was amplified by multiple displacement to comprehensively detect both DNA and RNA viruses [26]) and then analyzed by real-time PCR. For real-time PCR, we used the Ct numbers to show the richness of the virus. Viruses that were not detected were not shown. Primers were designed to amplify 
specific regions in the Bdellovibrio phage phiMH2K (5'AATCCTCAATTCCAGACTTCCA-3' (F) and 5'-CCAT TTCCATAAGTCCGAGTG-3' (R)), Bacillus phage B103 (5' - TGGCGATGTTGATGATGAC-3' (F) and 5'-CTTT ATTTGCGTCTGTTGTCG-3' (R)), columbid circovirus (5'-TCAGGAGACGAAGGACACG-3' (F) and 5'- TGGC ATCATACATCGGGAC-3' (R)), potato virus M (5'-CGCT TCGCTGCTTTCG - 3' (F) and 5'-CGGACCATTCATAC CACCA-3' (R)), Marseillevirus marseillevirus (5'-AAAGTC CCAAGTTATCACAAGC-3' (F) and 5' - TTTCTCGCAG CGTCAATG-3' (R)), simian sapelovirus (5'- TTCCATCT GCTCTAAATGCTCA-3' (F) and 5'-CAGCAGTTAGAG CGGGTG-3' (R)), and Andean potato mild mosaic virus (5'-AAGCCCAACATCGTTCTCC-3' (F) and 5' - AAGA GGATACGGGAGAAAGG-3' (R)).

\section{Redundancy analysis (RDA)}

Redundancy analysis (RDA) shows the interactions between sample distribution and environmental factors. We used vegan's RDA analysis in $\mathrm{R}$ with the phylumlevel abundances of the bacterial microbiome as environmental factors.

\section{Regression analysis}

We ran a regression analysis between bacterial microbiome diversity and virome richness with the stats package and plotted the results using the ggplot2 package.

\section{Metabolome detection}

The stool samples were suspended in methanol: $\mathrm{H}_{2} \mathrm{O}$ (4:1), ground, ultrasonicated, concentrated and dried so that the metabolome could be analyzed by GC-MS and LC-MS.

\section{GC-MS}

The derivatized samples were analyzed on an Agilent 7890A gas chromatography system coupled to an Agilent 5975C MSD system (Agilent). An HP-5 MS fusedsilica capillary column $(30 \mathrm{~mm} \times 0.25 \mathrm{~mm} \times 0.25 \mu \mathrm{m}$, Agilent) was utilized to separate the derivatives. Helium (>99.999\%) was used as the carrier gas at a constant flow rate of $6.0 \mathrm{~mL} / \mathrm{min}$ through the column. The injector temperature was maintained at $280^{\circ} \mathrm{C}$. A volume of $1 \mu \mathrm{L}$ was injected in splitless mode. The oven temperature was initially $60^{\circ} \mathrm{C}$ and was then ramped up to $125^{\circ} \mathrm{C}$ at a rate of $8{ }^{\circ} \mathrm{C} / \mathrm{min}$, to $190^{\circ} \mathrm{C}$ at a rate of $10^{\circ} \mathrm{C} / \mathrm{min}$, to $210^{\circ} \mathrm{C}$ at a rate of $4{ }^{\circ} \mathrm{C} / \mathrm{min}$ and to $310^{\circ} \mathrm{C}$ at a rate of $20^{\circ} \mathrm{C} / \mathrm{min}$; finally, the temperature was held at $310^{\circ} \mathrm{C}$ for $8.5 \mathrm{~min}$. The temperatures of the MS quadrupole and ion source (electron impact) were set to $150^{\circ} \mathrm{C}$ and $230^{\circ} \mathrm{C}$, respectively. The collision energy was $70 \mathrm{eV}$. Mass data were acquired in full-scan mode $(\mathrm{m} / \mathrm{z}$ 50-600), and the solvent delay time was set to $5 \mathrm{~min}$. The acquired MS data from GC-MS were analyzed by
ChromaTOF software (v 4.34, LECO, St Joseph, MI). Metabolites were qualitatively assessed by the Fiehn database, which is linked to ChromaTOF software. Briefly, after alignment with the Statistic Compare component, a CSV file was obtained with three-dimensional data sets, including sample information, peak name, retention time, $\mathrm{m} / \mathrm{z}$ and peak intensities. The resulting data were normalized to the total peak area of each sample in Excel 2007 (Microsoft, USA) and imported into SIMCA (version 14.0, Umetrics, Umeå, Sweden) to define the $95 \%$ confidence interval of the modeled variation. The differential metabolites were selected on the basis of the combination of a statistically significant threshold of variable influence on projection (VIP) values obtained from the OPLS-DA model and $p$ values from a two-tailed Student's t-test on the normalized peak areas, where metabolites with VIP values larger than 1.0 and $p$ values less than 0.05 were included.

\section{LC-MS}

LC-MS was performed on an Ultimate 3000-Velos Pro system equipped with a binary solvent delivery manager and a sample manager coupled with an LTQ Orbitrap mass spectrometer equipped with an electrospray interface (Thermo Fisher Scientific); an Acquity BEH C18 column $(100 \mathrm{~mm} \times 2.1 \mathrm{~mm}$ i.d., $1.7 \mu \mathrm{m}$; Waters $)$ was used. The column was maintained at $45^{\circ} \mathrm{C}$, and separation was achieved using the following gradient: $5 \% \mathrm{~B}-25 \% \mathrm{~B}$ from 0 to $1.5 \mathrm{~min}, 25 \% \mathrm{~B}-100 \% \mathrm{~B}$ from 1.5 to $10.0 \mathrm{~min}, 100 \% \mathrm{~B}$ from 10.0 to $13.0 \mathrm{~min}$; $100 \% \mathrm{~B}-5 \% \mathrm{~B}$ from 13.0 to 13.5 $\mathrm{min}$, and $5 \% \mathrm{~B}$ from 13.5 to $14.5 \mathrm{~min}$ at a flow rate of 0.40 $\mathrm{mL} / \mathrm{min}$, where B was acetonitrile $(0.1 \%$ (v/v) formic acid), and $\mathrm{A}$ was aqueous formic acid $(0.1 \%(\mathrm{v} / \mathrm{v})$ formic acid). The injection volume was $3.00 \mu \mathrm{L}$, and the column temperature was set at $45.0^{\circ} \mathrm{C}$. The mass spectrometric data were collected using an LTQ Orbitrap mass spectrometer equipped with an electrospray ionization (ESI) source operating in either positive or negative ion mode. The capillary and source temperatures were set at $350{ }^{\circ} \mathrm{C}$, with a desolvation gas flow of $45 \mathrm{~L} / \mathrm{h}$. Centroid data were collected from 50 to $1000 \mathrm{~m} / \mathrm{z}$ with a resolution of 30,000. XCMS (http://masspec.scripps.edu/ xcms/xcms.php) was used for nonlinear alignment of time domain data and automatic integration and extraction of the peak intensities. Default XCMS parameter settings were used (major default parameters: profmethod $=$ bin; method $=$ matched filter; step $=0.1)$ except for full width at half maximum $=$ 8 , bandwidth $(\mathrm{bw})=6$ and snthresh $=5$. Variables with $<$ $30 \%$ relative standard deviation (RSD) in QC samples were then retained for further multivariate data analysis. The result was a three-dimensional matrix that included retention time and $\mathrm{m} / \mathrm{z}$ pairs (variable indices), sample names (observations), and normalized ion intensities (variables). The positive and negative data were merged into a 
combined data set, which was imported into SIMCA-P+ 14.0 software (Umetrics, Umeå, Sweden). The differential metabolites were selected on the basis of a combination of statistically significant VIP values obtained from the OPLS-DA model and $p$ values from a two-tailed Student's t-test on the normalized peak areas, where metabolites with VIP values larger than 1.0 and $p$ values less than 0.05 were included. The differential metabolites were qualitatively assessed using the Human Metabolome Database (http://www.hmdb.ca/) and METLIN (https://metlin. scripps.edu/).

\section{Results}

We performed metagenomic sequencing of fecal samples to detect the bacterial microbiome and virome composition of healthy one-year-old rhesus monkeys housed at the IMBCAMS (Fig. 1). The rhesus monkeys were monitored by blood cell analysis, which is the examination of blood condition and disease by observing the number and distribution of blood cells during the course of antibiotic treatment [32], and we found no obvious differences between the normal monkeys and those treated with antibiotics (Additional file 1: Figure S1).

\section{The bacterial microbiome was depleted stably and continuously by antibiotic treatment}

The composition of the bacterial microbiome was investigated by extracting DNA directly from feces for $16 \mathrm{~S}$ rRNA gene amplification. We used the hypervariable regions V3 and V4 to perform phylogenetic discrimination with the barcode primers 338F/806R [27]. In total, 556,
012 amplicon reads $(37,067 \pm 6872$ per sample) were obtained.

At the phylum level, the fecal bacterial communities were composed predominantly of high abundances of Bacteroidetes (53.1\%) and Firmicutes (42\%) and low levels of Proteobacteria (3.82\%) (Additional file 2: Figure S2A). As expected, the enteric bacterial microbiome was depleted significantly after exposure to antibiotics (Fig. 2, 3a; Additional file 2: Figure S2, Additional file 3: Table S1, Additional file 4: Figure S3). The levels of Firmicutes and Bacteroidetes decreased to $7.69 \%$ and below $0.01 \%$, respectively; at the same time, the abundance of Proteobacteria increased to 92\% (Additional file 2: Figure S2B). Escherichia-Shigella were the major constituents of Proteobacteria, and other genera belonging to Proteobacteria were markedly depleted (Additional file 2 : Figure S2B, Additional file 3: Table S1). The fecal samples were spread on plates with the antibiotic cocktail, and a large number of colony-forming units (CFUs) were observed in the sample from D9 but not in the sample from D0. The $16 \mathrm{~S}$ rRNA amplicons of single clones were sequenced, and we found that these clones belonged to Escherichia-Shigella (data not shown). As expected, the overall diversity and richness of the bacterial microbiome were depleted both stably and continuously (Fig. 3 b, c). The Shannon diversity index showed that the diversity of the bacterial microbiome was significantly decreased after treatment with antibiotics and remained at a low level (Fig. 3B, Additional file 2: Figure S2C). The richness of the bacterial microbiome was significantly decreased, as measured by the ACE (Fig. 3c, Additional file 2: Figure S2D). In addition,

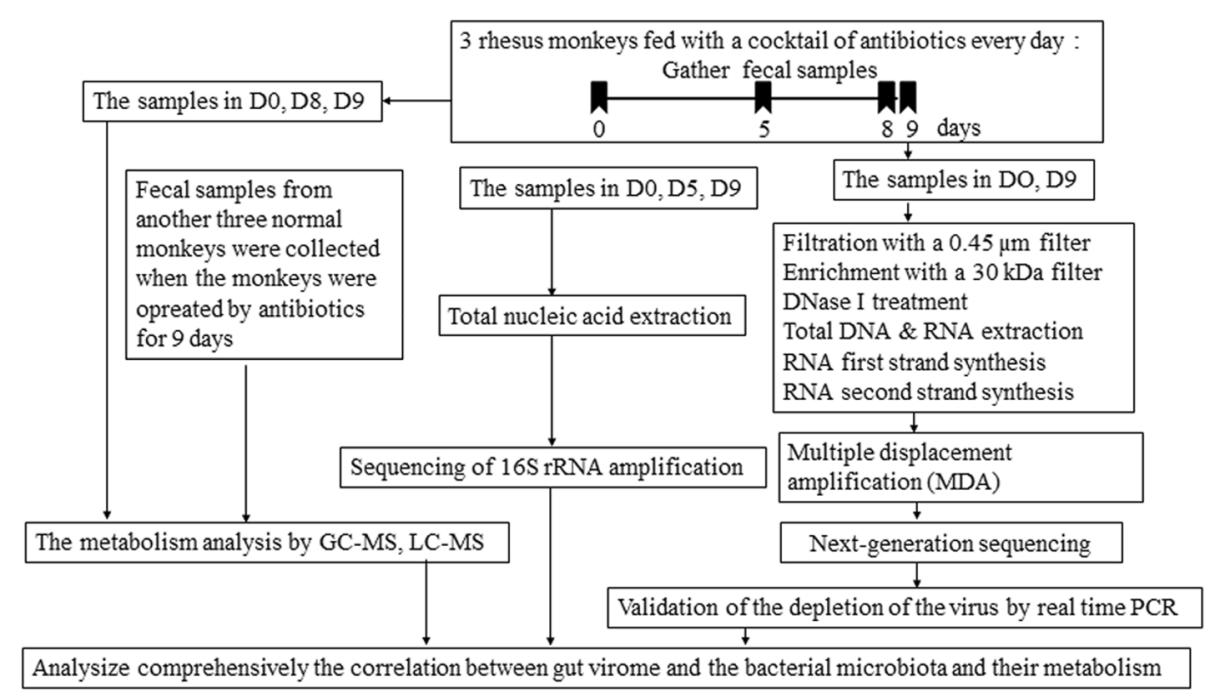

Fig. 1 Experimental procedure. Three rhesus monkeys were treated with an antibiotic cocktail to control their gut bacterial microbiome, and we detected the longitudinal changes in the gut bacterial microbiome at D0, D5 and D9 by 165 rRNA amplicon sequencing. Then, we extracted nucleic acids from the fecal supernatant at D0 and D9 and scanned the gut viromes of the monkeys. The samples for metabolomics were collected on D0, D8 and D9 and scanned by GC-MS and LC-MS. We comprehensively analyzed the interactions among the gut virome, bacterial microbiome and metabolomes based on the above results 


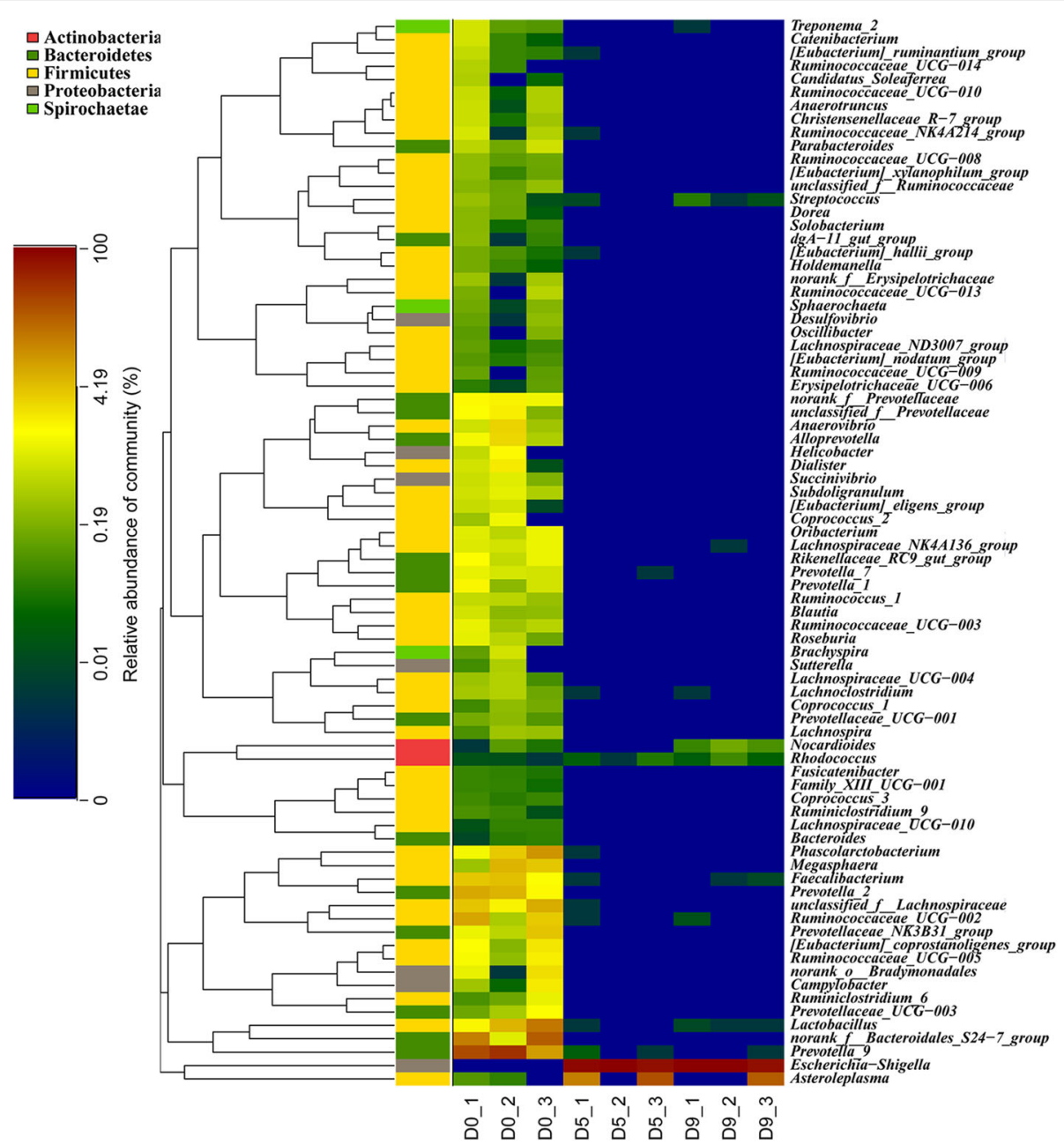

Fig. 2 The bacterial microbiome was obviously depleted by treatment with antibiotics. Heatmap of the OUT percentage of every genus at 3 timepoints. Each point represents 3 biological replicates. The genera that belong to the same phylum are shown in the same color on the left. The total OTU number of 3 biological replicates for every genus was more than 10 . The color bar represents the log of the percentage, the numbers in the heatmap are the log values of the OTU numbers, and the numbers in the bar are the percentages

there were noticeable differences in bacterial $\beta$-diversity between control and experimental animals, as determined using principal component analysis (PCA), and the results showed good repeatability within a single group (Additional file 2: Figure S2F).

Together, these data suggest that the richness and diversity of the bacterial microbiome composition were depleted stably and continuously. Therefore, we assessed the virome composition in the control and antibiotictreated experimental monkeys on the ninth day.

The virome community composition changed noticeably after treatment with antibiotics

In previous experiments, examination of microbiota genomes from rhesus macaques (Macaca mulatta) showed that a majority of the sequences in the fecal samples were mapped to bacterial genomes, while the percentage of sequences mapped to viral genomes was very low [20]. To comprehensively detect both DNA and RNA viruses, we filtered the fecal samples with a $0.45-\mu \mathrm{m}$ filter and treated the samples with DNase I, after which, total DNA and RNA were extracted separately from the same fecal sample. Owing to low yield, amplification of the DNA and double-stranded cDNA by whole-genome amplification (using MDA) was necessary. Using the MiSeq $2 \times 250$ paired-end protocol on the Illumina MiSeq platform, we obtained an average of 54,590,439 \pm $14,898,536$ clean reads per MDA sample library and generated a total of $88,610 \mathrm{Mbp}$ from 12 samples, allowing detailed investigation of the viral populations. To catalog the present genes, we predicted open reading frames (ORFs). A total of 478,694 ORFs were predicted; the average ORF length was $398.1603279 \mathrm{bp}$, with a maximum of $16,635 \mathrm{bp}$ and a minimum of $100 \mathrm{bp}$. The 

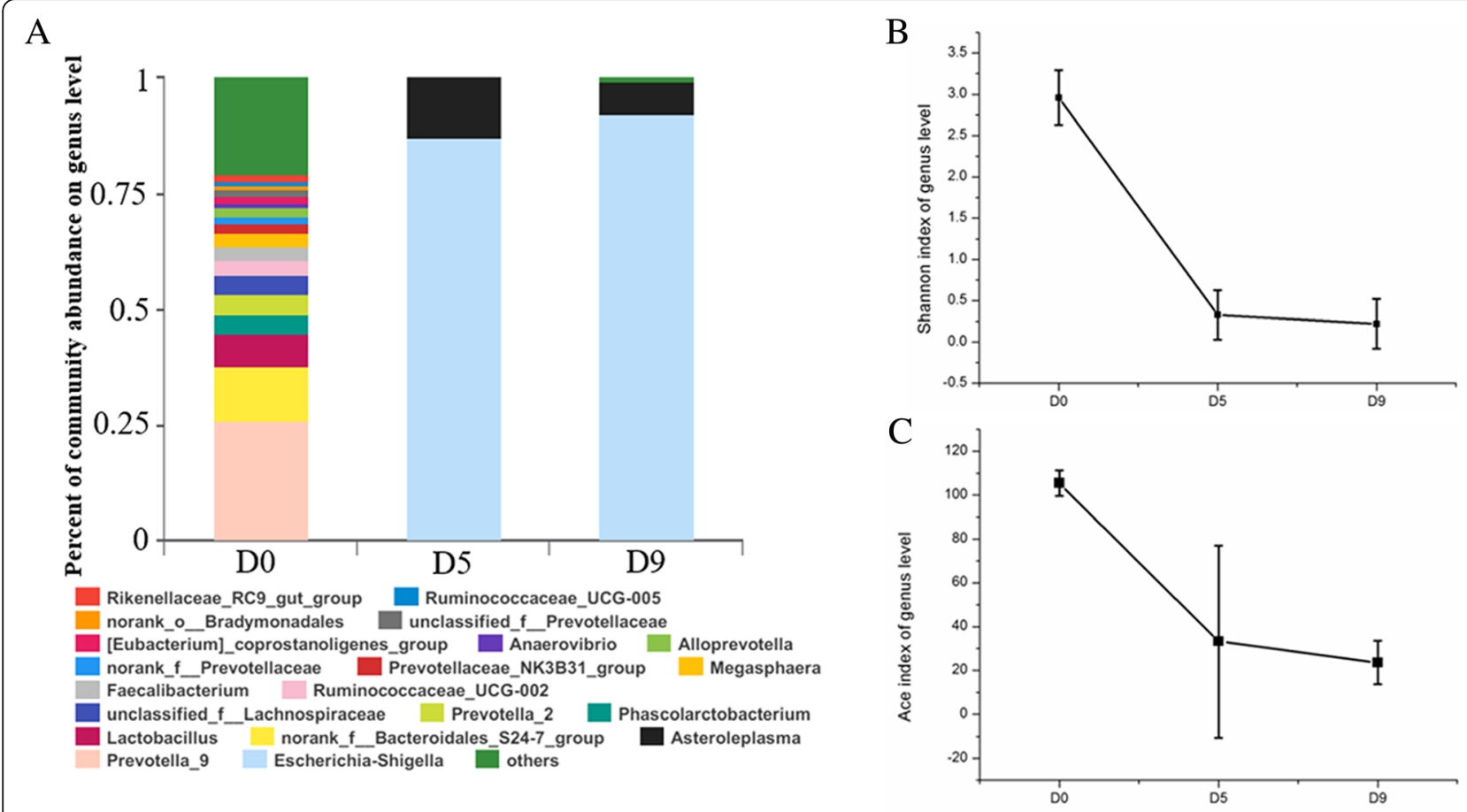

Fig. 3 The bacterial microbiome was depleted stably and continuously by treatment with antibiotics. (a) Percentage of community abundance at the genus level at each timepoint. The bacterial composition changed noticeably, and the diversity of the bacterial microbiome decreased sharply. We took the average of the 3 biological replicates at each timepoint, and every genus is presented in its own color. (b, $\mathbf{c})$ a-Diversity analysis of the bacterial microbiome calculated by Mothur (version v.1.30.1). The Shannon diversity index represents the diversity of the bacterial microbiome, and the ACE shows richness. We show the average of the 3 biological replicates at each timepoint

ecological signatures of the intestinal virome have been characterized. The largest percentage of sequences mapped to viromes in the fecal samples belonged to bacteriophages, accounting for over $80 \%$ of the sequences (Additional file 5: Table S2). We also identified eukaryotic DNA viruses and RNA viruses, as well as other viral families that are defined as "unclassified" in the NCBI Taxonomy database. In the raw data from the virome metagenomic analysis, RNA virus sequences were found in the DNA virus results, and DNA virus sequences were found in the RNA virus results, because the RNA virus sequences were wrongly affiliated with DNA viral genomes and vice versa. In addition, DNA viruses, especially bacteriophages, were the main components of the virome in our sequencing data, and the RNA from these viruses may have been extracted together with RNA viruses and vice versa. Thus, we excluded these data.

We observed that the fecal virome composition was noticeably altered after depletion of the bacterial microbiome treated with antibiotics. ANOSIM showed that the distance between groups was greater than that within groups for DNA viruses and bacteriophages (Additional file 6: Figure S4). DNA viruses, including members of the families Poxviridae, Iridoviridae, Ascoviridae, Baculoviridae, Marseilleviridae, and Mimiviridae and bacteriophages, such as members of the family Inoviridae, were present in the normal monkeys but absent after the gut bacterial microbiome was depleted (Fig. 4). DNA viruses, including members of the families Herpesviridae, Nanoviridae, and Phycodnaviridae, were present in three biological replicates before antibiotic treatment but in only one biological replicate after the gut bacterial microbiome was depleted (Fig. 4). Most of the reads from bacteriophages were noticeably depleted after the gut bacterial microbiome was depleted (Additional file 7: Figure S5, Additional file 8: Table S3). RNA viruses, including members of the families Picornaviridae and Tymoviridae, were present in three biological replicates but were present in only one biological replicate after the gut bacterial microbiome was depleted, and RNA viruses belonging to Nodaviridae were present in two biological replicates but absent after the gut bacterial microbiome was depleted (Fig. 4). In addition, many kinds of viral groups, including Circoviridae, Geminiviridae, Microviridae, Podoviridae, Myoviridae, Siphoviridae, Picornaviridae, and Retroviridae, were present regardless of whether the bacterial microbiota was depleted, but the sequencing reads showed that the abundances of these viruses may have decreased with bacterial microbiota depletion (Additional file 7: Figure S5). However, we could not validate this decrease 


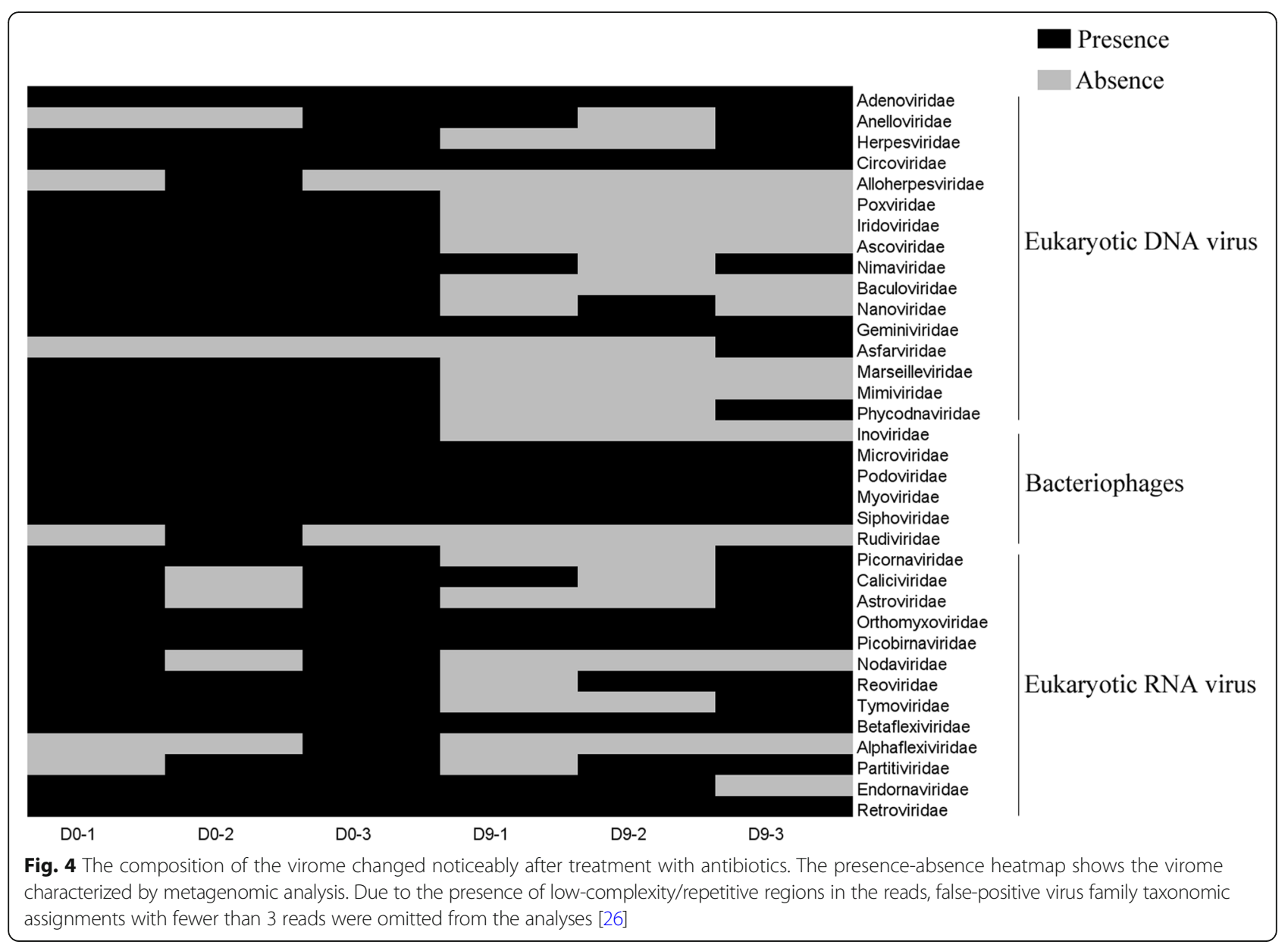

because the data from deep sequencing were only qualitative, not quantitative.

\section{Depletion of the virome at the species level was validated} by real-time PCR

Regarding the abundances of viruses at the species level, the results that were similar in 2 or more monkeys were selected, and we used real-time PCR to validate changes in the richness of the viruses. As the figure shows, we analyzed fecal samples that had not been subjected to MDA. After the bacterial microbiome was depleted, the results from real-time PCR validated the depletion of 4 viral species: Bdellovibrio phage phiMH2K, which belongs to the Microviridae family of bacteriophages; Bacillus phage B103, which belongs to the Podoviridae family of bacteriophages; columbid circovirus, which belongs to the Circoviridae family of DNA viruses; and potato virus $M$, which belongs to the Betaflexiviridae family of RNA viruses (Fig. 5a). The samples that were not detected directly from DNA or cDNA were subjected to MDA and then detected by real-time PCR. The depletion of 3 viral species was detected (Fig. 5b): Marseillevirus marseillevirus, which belongs to the Marseilleviridae family of DNA viruses; simian sapelovirus, which belongs to the
Picornaviridae family of RNA viruses; and Andean potato mild mosaic virus, which belongs to the Tymoviridae family of RNA viruses. In addition, Mason-Pfizer monkey virus, which belongs to the Retroviridae family, was detected among the RNA viruses; this virus is very dangerous in monkey populations and has been shown to cause an AIDS-like disease in rhesus macaques [33]. Encouragingly, no MasonPfizer monkey virus was detected by real-time PCR.

Briefly, the fecal virome composition was noticeably altered after depletion of the bacterial microbiome, and the abundances of many DNA viruses, bacteriophages and RNA viruses in the gut were clearly decreased. In addition, in the metagenomic analysis, we found high numbers of reads from DNA viruses and bacteriophages; however, low numbers of reads from RNA viruses were found (Additional file 7: Figure S5, Additional file 8: Table S3). These results may be due to the limited application of MDA in RNA viruses.

Shifts in the virome were correlated with deletion of the bacterial microbiome

The microbiota structure is the result of dynamic interactions among various community members. We found 


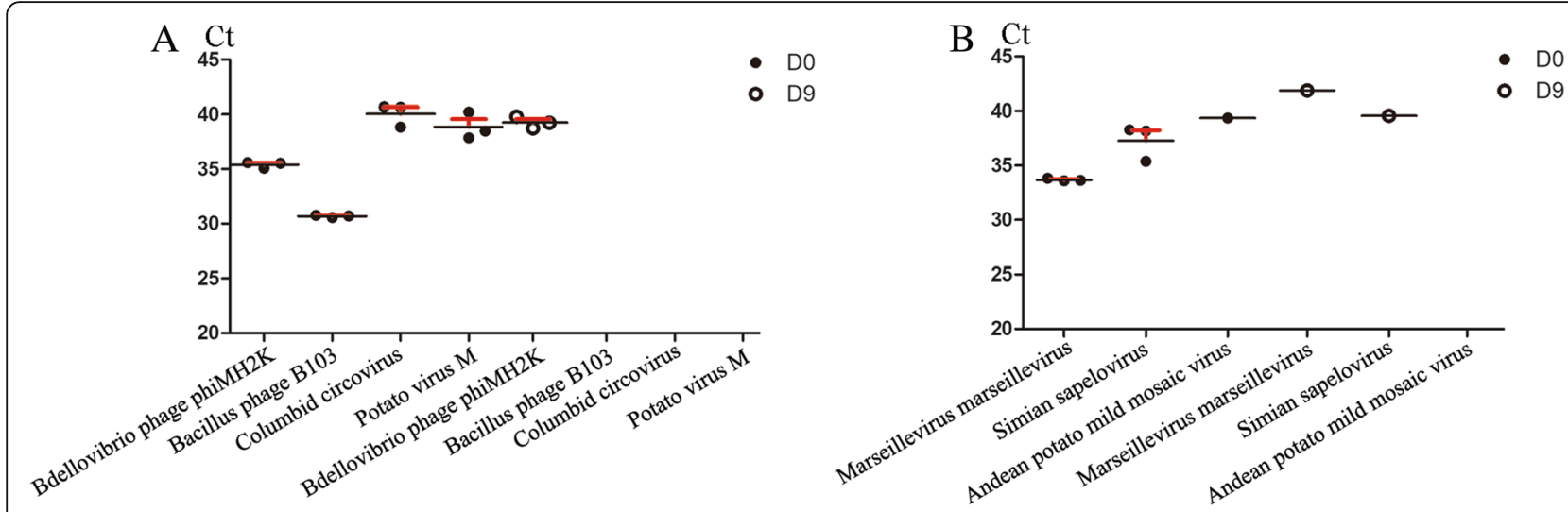

Fig. 5 Depletion of viromes at the species level after depletion of the gut bacterial composition validated by real-time PCR. (a) As the templates, the DNA and CDNA extracted from fecal samples were detected directly by real-time PCR. (b) The samples that were not detected directly from DNA or CDNA were subjected to MDA and then used as templates for detection by real-time PCR. We used the Ct numbers to show viral richness. The black line represents the mean of 3 replicates, and the red line represents the SEM. The samples that were not detected are not shown

a close interaction between the whole virome (DNA viruses, RNA viruses and bacteriophages) and the bacterial microbiome. We analyzed the effects on the abundance of the virome by RDA, taking the richness of bacteria at the phylum level as environmental factors, and found a negative interaction between the abundances of DNA viruses and bacteriophages at D9 and the abundances of most bacteria (Fig. 6a, b). However, RNA viruses exhibited chaotic interactions due to weak repeatability. Next, we conducted linear regression analysis between virome abundance and both the ACE and Shannon diversity index, and we found positive correlations in bacteriophages (Fig. 6c, d). In addition, the DNA and RNA viruses showed a positive trend (Additional file 9: Figure S6) but weak confidence levels.

Overall, these results support our hypothesis that a clear interomic relationship exists between the virome and bacterial microbiome. Positive correlations were found between virome abundance and the richness and diversity of the bacterial microbiome.

\section{Metabolites produced by the bacterial microbiome shifted noticeably and could inhibit or promote the survival of viruses}

Metabolites could inhibit or promote viruses in vivo [34] and in vitro [35-37], and the metabolites produced by bacteria play important roles in host physiology [38]. To interrogate the functions associated with the response to depletion in the bacterial microbiome, we performed a KEGG prediction analysis of the bacterial microbiome using PICRUSt. We observed significant differences in functional systems along with shifts in the composition of the bacterial microbiome, according to the predictions by PICRUSt. KEGG pathways associated with bacterial toxins were downregulated significantly (Fig. 7a), perhaps as a result of antibiotic treatment. The pathway associated with D-arginine metabolism showed a 9-fold decrease; in contrast, the pathways associated with fatty acid metabolism and tryptophan metabolism showed a 2-fold and 2.6-fold increase, respectively. At the same time, the biosynthetic pathway for ubiquinone and other terpenoidquinones showed a 2-fold increase. Moreover, the glycosaminoglycan degradation pathway exhibited low diversity and a 1300-fold decrease (Fig. 7a). Glycan [39], glycosaminoglycan [40], quinone [41] and arginine $[42,43]$ are well known to support the inhibition of viruses, while tryptophan $[44,45]$ and fatty acids [36] promote viral survival.

Interestingly, we detected the metabolites in the fecal samples by metabolome scanning and found that the changes in some metabolite levels were consistent with our prediction based on the normalization of OTUs in the $16 \mathrm{~S}$ rRNA amplicon sequencing data. Because metabolomic analysis requires 6 biological duplications, the metabolomes of 6 samples collected before treatment with antibiotics and those of samples collected after treatment with antibiotics for 8 and 9 days were examined by GC-MS and LC-MS. The metabolomic results exhibited good repeatability (Additional file 10: Figure S7). In GC-MS (Fig. 7b), $\mathrm{N}$-dimethylarginine was not detected in monkeys that were not treated with antibiotics, but the metabolite was present after antibiotic treatment. The levels of $\mathrm{N}$-acetyltryptophan and $\mathrm{N}$-methyltryptophan showed a 3.3-fold and 1.85-fold decrease, respectively, after treatment with antibiotics. In LC-MS (Fig. 7c), we found that the levels of abruquinone $B$ and sulfated dihydromenaquinone-9 exhibited a 30-fold and 5.6fold increase, respectively. Regrettably, in the present study, we did not measure the levels of glycosaminoglycan, which plays a very important role in reducing the viral population [40]. 


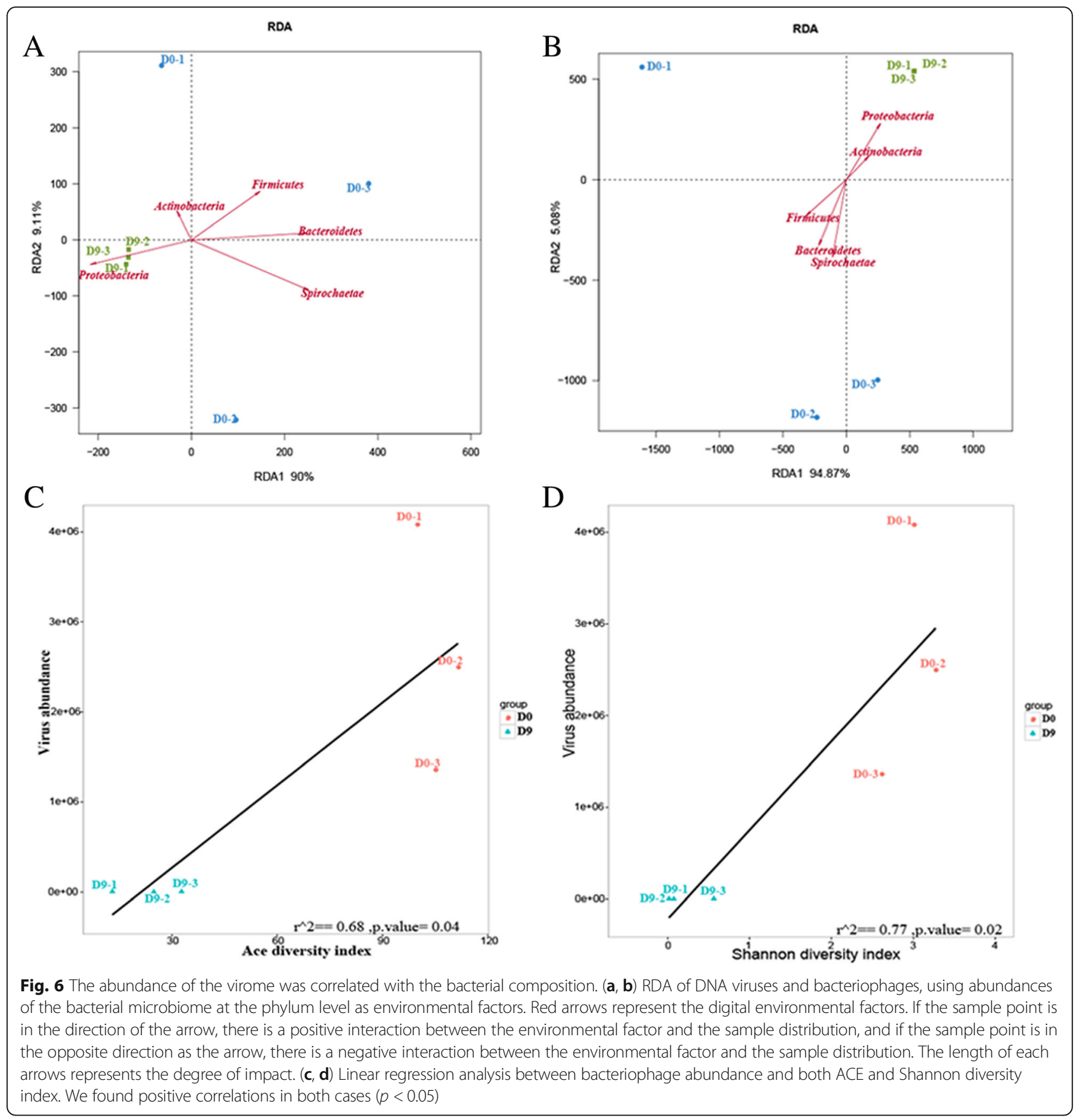

\section{Discussion}

As reported by Adina Howe, Yatsunenko $\mathrm{T}$ and Alejandro Reyes, in the same environment and feeding conditions, the composition of the microbiota and virome could remain stable within an individual [17, 46, 47]. However, the gut microbial composition could be influenced by multiple interacting factors, such as diet [46], antibiotic use [48], age, geographical setting [47], and several diseases, including chronic inflammation, obesity and diabetes [4]. In our study, the major reason for depletion of the gut bacterial microbiota was treatment with the antibiotic cocktail. The feeding conditions of the rhesus monkeys were stable in terms of their food and water consumption, and blood samples were monitored routinely, showing that there was no infection during the study period (Additional file 1: Figure S1). The bacterial composition exhibited stable and continuous depletion after treatment with the antibiotic cocktail, and we found that the virome composition changed noticeably and was correlated with the shifts in the bacterial community. Moreover, we found that metabolites produced by the gut bacterial microbiome may play a 


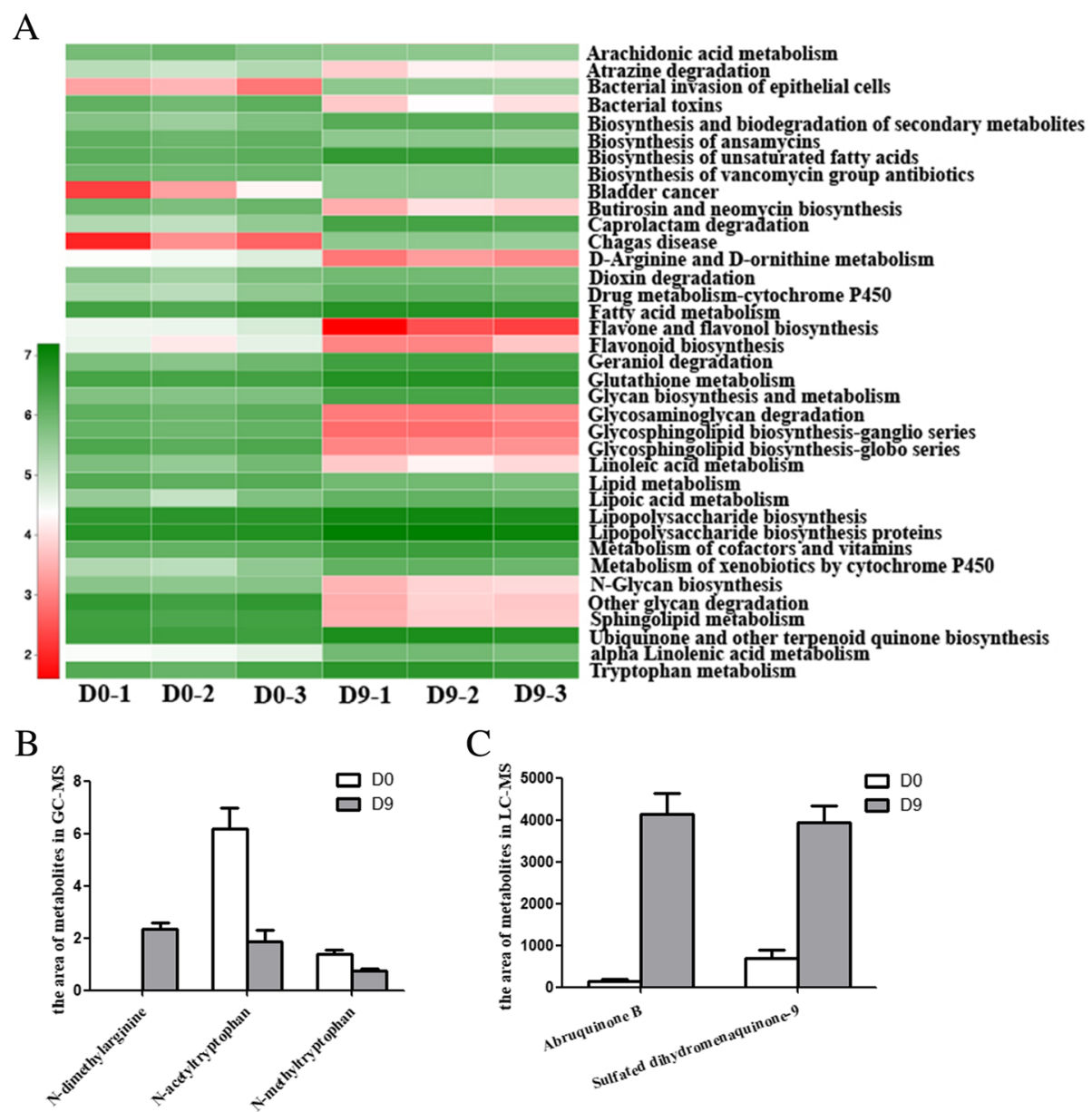

Fig. 7 Metabolites produced by the bacterial microbiome shifted noticeably and could inhibit or promote the survival of viruses. (a) Heatmap of the richness of metabolites in KEGG predicted by normalization of OTUs in the bacterial microbiome using PICRUSt. The numbers in the heatmap are the log values of metabolite richness. (b, c) The metabolites detected by LC-MS and GC-MS were consistent with the predictions from the KEGG analysis. The number represents the integral value of the peak area

role in the interrelation. In addition, we found that the composition of the rhesus monkey enterovirus group was similar to that of the human enterovirus group [26], and our results may be beneficial for research on the composition of the human virome.

When the bacterial microbiome was depleted, ampicillin could kill most bacteria, including gram-positive and gram-negative bacteria; streptomycin could kill most bacilli; kanamycin could kill most gram-negative bacteria; metronidazole could kill most anaerobic bacteria and parasites; and vancomycin could kill most gram-positive bacteria. Of course, the numbers of drug-resistant bacteria are increasing, but we believe that the cocktail of five antibiotics could deplete most of the commensal bacteria in the gut. As expected, the whole gut bacterial microbiome, including gram-positive and gram-negative bacteria (Additional file 2: Figure S2E), was depleted after treatment with the antibiotic cocktail, except for Escherichia-Shigella species belonging to Proteobacteria, which were resistant to the cocktail. Escherichia harbored the most diverse antibiotic resistance genes, including genes resistant to multidrug treatments, tetracycline, aminoglycoside, macrolide-lincosamidestreptogramin B, $\beta$-lactams, and sulfonamides [49]. We maintained the bacteria belonging to Escherichia-Shigella in plates with the antibiotic cocktail. In the future, we will investigate the specific resistance and antibiotic resistance genes in this bacterium. Notably, our study focused on the interaction between virome composition and the bacterial microbiome in rhesus monkeys and may serve as a model for gut microbiota analysis. Therefore, we used the administration of 5 distinct antibiotics at high dosages and high frequencies for 2 weeks to deplete the whole gut bacterial microbiome. In our results, the richness and diversity of the bacterial community were depleted. Because our study did not involve clinical treatment, the normal dose of antibiotics was not evaluated by our procedure. 
People are widely prescribed antibiotics each year [50], and while antibiotics exert very complex effects on the whole bacterial microbiome [48], the effects of these drugs on the virome are not clear. Antibiotics can directly affect viruses but do not exhibit a wide range of roles. Minocycline [51], berberine, abamectin, ivermectin [52], glycopeptides [53], and teicoplanin [54] could inhibit the corresponding viruses. In our study design, an antibiotic cocktail that included ampicillin, streptomycin, kanamycin, metronidazole, and vancomycin was administered orally. No study has yet reported that these antibiotics directly affect viruses.

Based on our results, the richness of these viruses was very low in the gut, and we had to use MDA to perform deep sequencing, although the detection by deep sequencing was very sensitive. We first characterized the shift in the gut virome by deep sequencing, and the samples were amplified by MDA. MDA is used as a general technique in virome research, especially for DNA virome detection [26]. To a certain extent, the amplification read-out can also represent the virus quantity. However, MDA is not well suited to the detection of RNA viruses. The sequence-independent amplification (SIA) approach is more appropriate than MDA for detecting RNA viruses [55]. In the future, we can use this approach to precisely detect RNA viruses. In this case, we validated the depletion of the virome composition, including DNA viruses, RNA viruses and bacteriophages, by real-time PCR. Although the number of cycles seemed high, these results were verified via three biological replicates, and the results of the no-template control (NTC) were not detected. In addition, we performed serial dilution of the in vitro transcribed RNA of coxsackievirus A16 to generate a standard curve and found that a Ct of 39.96 represents 23 genomic copies (data not shown). In our opinion, these viruses are components of the gut microbiome with low richness and may be involved in host physiology.

The metabolites produced by gut bacteria play very important roles in host physiology [38], although the effects of these metabolites on virome composition have rarely been reported. Glycan [39], glycosaminoglycan [40], quinone [41] and arginine [42, 43] support the inhibition of viruses, while tryptophan $[44,45]$ and fatty acids [36] promote viral survival. Although most metabolites that can inhibit or promote viruses play roles in human viruses, tryptophan could promote the simian immunodeficiency virus in macaques [45]. In addition, most pandemics originating in animals, such as severe acute respiratory syndrome and pandemic influenza, could start to appear because of ecological, behavioral, or socioeconomic changes [56]. Many human viruses are zoonotic, and some human viruses, such as human enterovirus 71, can infect animals, especially monkeys [32].
We believe that metabolites play roles in a broad spectrum of viruses and that changes in the metabolites may correlate with depletion of the virome. In our results, the level of quinone, which decreases the abundance of viruses, was increased in the gut metabolome, and the levels of some amino acids that promote the survival of viruses, such as tryptophan, were decreased. Importantly, glycosaminoglycan, which can reduce the populations of various viruses, was noticeably increased in the KEGG pathways of the bacterial microbiome, but we did not measure glycosaminoglycan levels in the present study. It is very difficult to detect glycosaminoglycan by metabolic scanning because glycosaminoglycan has a very high molecular weight. In the future, glycosaminoglycan levels could be measured by time-offlight mass spectrometry. First, the polysaccharide needs to be dispelled, followed by detection of the monosaccharide to calculate the polysaccharide levels based on the relationships among the monosaccharides in a specific database. However, this process is very complicated, and the database is not sufficiently large at present. By analyzing the relevant data, we found that depletion of bacteria directly promoted changes in the concentrations of some metabolites, which may play important roles in reducing the abundance of DNA viruses.

\section{Conclusion}

Our metagenomic-scale characterization of the virome composition after treatment with antibiotics supports the notion that the composition of the virome is noticeably altered in correlation with bacterial community depletion and that metabolites produced by bacteria possibly play important roles in the interaction. The next step will be to investigate the underlying mechanisms in detail.

\section{Additional files}

Additional file 1: Figure S1. The detection of blood cell analysis during the course of antibiotic treatment. White blood cells (WBC), neutrophilic granulocytes (NEUT), lymphocytes (LYMPH), monocytes (MONO\#), eosinophils (EO\#), and basophilic granulocytes (BASO\#) were counted, and the counts were compared between the monkeys that were treated with antibiotics and ones were not, and there was no obvious difference. (TIF $597 \mathrm{~kb}$ )

Additional file 2: Figure S2. The richness and diversity of gut bacterial microbiota were depleted obviously stably and continuously. (A, B) The community analysis of gut bacterial microbiota on phylum level. The phylum was represented by own color.(C, D) The student's t-test of Alpha diversity index (the Shannon diversity index and the ACE estimator) in genus level. $0.01<P \leq 0.05$ was marked ${ }^{*}, 0.001<P \leq 0.01$ was marked * ${ }^{*}, P \leq 0.001$ was marked ***. (E)The longitudinally reads of OTU in grampositive and gram-negative bacteria. (F) The repeatability analysis of $16 \mathrm{~S}$ rRNA amplicon sequencing by PCA. (TIF $978 \mathrm{~kb}$ )

Additional file 3: Table $\mathbf{S}$ 1. The reads number on the genus level in the gut bacterial communities in a longitudinal cohort treated with an antibiotic cocktail. (XLS $22 \mathrm{~kb}$ ) 
Additional file 4: Figure S3. The phylogenetic tree on genus level of gut bacterial microbiome. The number in the line represents the genetic distance. Every phlym was showed in own color. The bar in the right were caculated according to the number of reads. (TIF $966 \mathrm{~kb}$ )

Additional file 5: Table S2. The reads number on the family level in the gut virome communities in a longitudinal cohort treated with an antibiotic cocktail. (XLS $2 \mathrm{~kb}$ )

Additional file 6: Figure S4. The Anosim analysis of virome groups. The ordinate represents the distance value. $R$ value represents the statistic results, and the closer the $R$ value is to 1 , the greater the difference between groups than the difference in the group, and the grouping was reasonable. (TIF $495 \mathrm{~kb}$ )

Additional file 7: Figure S5 (A) Heatmap of abundance of the DNA virus composition on the family level. (B) Heatmap of abundance of the bacteriophages composition on the family level. (C) Heatmap of abundance of the RNA virus composition on the family level. The hosts that belong to the same domain are shown in the same color on the left. The abundance were represented by the summation of the reads number and the contigs numbers which removed the repeated number with corresponding reads, and the color bar showed the summation gradient. The results which were similar in 2 and more than 2 monkeys were analyzed in our results. (TIF $723 \mathrm{~kb}$ )

Additional file 8: Table S3. The reads number on the family level belong to bacteriophages and the eukaryotic viruses including DNA viruses and RNA viruses. (XLS $27 \mathrm{~kb}$ )

Additional file 9: Figure S6. The linear regression analysis between virome abundance and Ace estimator index, the Shannon diversity index. (A, B) The linear regression analysis between DNA virome abundance and Ace estimator index, Shannon diversity index. (C, D) The linear regression analysis between RNA virome abundance and Ace estimator index, Shannon diversity index. (TIF $441 \mathrm{~kb}$ )

Additional file 10: Figure S7. The Principal Component Analysis analysis of metabolome detecting by PCA. (A) PCA analysis of GC-MS. (B) PCA analysis of LC-MS. (TIF $261 \mathrm{~kb}$ )

\section{Abbreviations}

ACE: Abundance-based coverage estimator; bp: Base pair; CFU: Colonyforming unit; COG: Cluster of Orthologous Groups of proteins; Ct: Cycle threshold; GC-MS: Gas chromatography-mass spectrometry; KEGG: Kyoto Encyclopedia of Genes and Genomes; KO: KEGG Ortholog; LC-MS: Liquid chromatography-mass spectrometry; MDA: Multiple displacement amplification; NTC: No-template control; ORF: Open reading frame; OTU: Operational taxonomic unit; PCA: Principal component analysis; PICRUSt: Phylogenetic investigation of communities by reconstruction of unobserved states; RDA: Redundancy analysis; SIA: Sequence-independent amplification

\section{Acknowledgments}

We thank Majorbio for their contributions to the 16S rRNA amplicon and virome sequencing projects.

\section{Authors' contributions}

LL, HS and HEL conceived the study and designed the experiments and scientific objectives. LL led the project. HS analyzed the data. HEL performed the experiments, analyzed the data and prepared the manuscript. $\mathrm{HOL}$ provided the materials and performed the experiments. JW, LG, HF, HZ, ZY, $\mathrm{XH}$ and $\mathrm{MC}$ contributed to the experiments. $\mathrm{FY}$ and $\mathrm{ZH}$ contributed to the feeding and maintenance of the animals. NL, JY and QW participated in discussions and manuscript preparation. All authors discussed the results and commented on the manuscript.

\section{Funding}

This work was supported by the CAMS Innovation Fund for Medical Sciences (2016-I2M-1-014; 2017-I2M-2-006), the Yunnan Applied Basic Research Projects (2018FB113) and the Yunnan innovation team in China (2016HC009).

\section{Availability of data and materials}

All data generated or analyzed during this study are included in this published article and the additional files. We have deposited the raw sequencing data into NCBI and the number is PRJNA555120.

\section{Ethics approval and consent to participate}

All animal experiments were conducted under prior approval from the Animal Ethics Committee of the Institute of Medical Biology, Chinese Academy of Medical Sciences, with permit number DWSP201803006, according to the National Guidelines on Animal Work in China.

\section{Consent for publication}

Not applicable.

\section{Competing interests}

The authors declare that they have no competing interests.

\section{Author details}

${ }^{1}$ Institute of Medical Biology, Chinese Academy of Medical Sciences \& Peking Union Medical College, Kunming 650118, China. ${ }^{2}$ Key Laboratory of Systemic Innovative Research on Virus Vaccine, Chinese Academy of Medical Sciences, Kunming, China.

Received: 24 April 2019 Accepted: 5 August 2019

Published online: 19 August 2019

\section{References}

1. Dahiya DK, Renuka, Puniya M, Shandilya UK, Dhewa T, Kumar N, Kumar S, Puniya AK, Shukla P: Gut Microbiota Modulation and Its Relationship with Obesity Using Prebiotic Fibers and Probiotics: A Review Front Microbiol 2017, 8:563.

2. Shreiner $A B, K a o J Y$, Young VB. The gut microbiome in health and in disease. Curr Opin Gastroenterol. 2015;31(1):69-75.

3. Konturek PC, Brzozowski T, Konturek SJ. Stress and the gut: pathophysiology, clinical consequences, diagnostic approach and treatment options. J Physiol Pharmacol. 2011;62(6):591-9.

4. Karlsson F, Tremaroli V, Nielsen J, Backhed F. Assessing the human gut microbiota in metabolic diseases. Diabetes. 2013;62(10):3341-9.

5. Turnbaugh PJ, Ley RE, Mahowald MA, Magrini V, Mardis ER, Gordon JI. An obesity-associated gut microbiome with increased capacity for energy harvest. Nature. 2006:444(7122):1027-31.

6. Hsiao EY, McBride SW, Hsien S, Sharon G, Hyde ER, McCue T, Codelli JA, Chow J, Reisman SE, Petrosino JF, et al. Microbiota modulate behavioral and physiological abnormalities associated with neurodevelopmental disorders. Cell. 2013;155(7):1451-63.

7. van Nood E, Vrieze A, Nieuwdorp M, Fuentes S, Zoetendal EG, de Vos WM Visser CE, Kuijper EJ, Bartelsman JF, Tijssen JG, et al. Duodenal infusion of donor feces for recurrent Clostridium difficile. N Engl J Med. 2013;368(5): 407-15

8. Kelly CR, Kahn S, Kashyap P, Laine L, Rubin D, Atreja A, Moore T, Wu G. Update on fecal microbiota transplantation 2015: indications, methodologies, mechanisms, and outlook. Gastroenterology. 2015;149(1): 223-37.

9. Minot S, Sinha R, Chen J, Li H, Keilbaugh SA, Wu GD, Lewis JD, Bushman FD. The human gut virome: inter-individual variation and dynamic response to diet. Genome Res. 2011;21(10):1616-25.

10. Reyes A, Semenkovich NP, Whiteson K, Rohwer F, Gordon Jl. Going viral: next-generation sequencing applied to phage populations in the human gut. Nat Rev Microbiol. 2012;10(9):607-17.

11. Duerkop BA, Clements CV, Rollins D, Rodrigues JL, Hooper LV. A composite bacteriophage alters colonization by an intestinal commensal bacterium. Proc Natl Acad Sci U S A. 2012;109(43):17621-6.

12. Barr JJ, Auro R, Furlan M, Whiteson KL, Erb ML, Pogliano J, Stotland A, Wolkowicz R, Cutting AS, Doran KS, et al. Bacteriophage adhering to mucus provide a non-host-derived immunity. Proc Natl Acad Sci U S A. 2013; 110(26):10771-6.

13. Barton ES, White DW, Cathelyn JS, Brett-McClellan KA, Engle M, Diamond MS, Miller VL, Virgin HW. Herpesvirus latency confers symbiotic protection from bacterial infection. Nature. 2007:447(7142):326-9.

14. Virgin HW. The virome in mammalian physiology and disease. Cell. 2014; 157(1):142-50. 
15. He Q, Gao Y, Jie Z, Yu X, Laursen JM, Xiao L, Li Y, Li L, Zhang F, Feng Q, et al. Two distinct metacommunities characterize the gut microbiota in Crohn's disease patients. Gigascience. 2017;6(7):1-11.

16. Chehoud C, Dryga A, Hwang Y, Nagy-Szakal D, Hollister EB, Luna RA, Versalovic J, Kellermayer R, Bushman FD. Transfer of viral communities between human individuals during fecal microbiota transplantation. MBio. 2016;7(2):e00322.

17. Reyes A, Haynes M, Hanson N, Angly FE, Heath AC, Rohwer F, Gordon Jl. Viruses in the faecal microbiota of monozygotic twins and their mothers. Nature. 2010;466(7304):334-8.

18. Siegel SJ, Roche AM, Weiser JN. Influenza promotes pneumococcal growth during coinfection by providing host sialylated substrates as a nutrient source. Cell Host Microbe. 2014;16(1):55-67.

19. Khan MN, Xu Q, Pichichero ME. Protection against Streptococcus pneumoniae Invasive Pathogenesis by a Protein-Based Vaccine Is Achieved by Suppression of Nasiopharyngeal Bacterial Density during Influenza A Virus Coinfection. Infect Immun. 2017;85(2):e00530-16.

20. Handley SA, Desai C, Zhao G, Droit L, Monaco CL, Schroeder AC, Nkolola JP, Norman ME, Miller AD, Wang D, et al. SIV infection-mediated changes in gastrointestinal bacterial microbiome and Virome are associated with immunodeficiency and prevented by vaccination. Cell Host Microbe. 2016; 19(3):323-35.

21. Deriu E, Boxx GM, He X, Pan C, Benavidez SD, Cen L, Rozengurt N, Shi W, Cheng G. Influenza virus affects intestinal microbiota and secondary Salmonella infection in the gut through type I interferons. PLoS Pathog. 2016;12(5):e1005572.

22. Jones MK, Watanabe M, Zhu S, Graves CL, Keyes LR, Grau KR, GonzalezHernandez MB, lovine NM, Wobus CE, Vinje J, et al. Enteric bacteria promote human and mouse norovirus infection of B cells. Science. 2014;346(6210): 755-9.

23. Karst SM. The influence of commensal bacteria on infection with enteric viruses. Nat Rev Microbiol. 2016;14(4):197-204.

24. Ichinohe T, Pang IK, Kumamoto Y, Peaper DR, Ho JH, Murray TS, Iwasaki A. Microbiota regulates immune defense against respiratory tract influenza a virus infection. Proc Natl Acad Sci U S A. 2011;108(13):5354-9.

25. Reyes A, Wu M, McNulty NP, Rohwer FL, Gordon Jl. Gnotobiotic mouse model of phage-bacterial host dynamics in the human gut. Proc Natl Acad Sci U S A. 2013;110(50):20236-41.

26. Lim ES, Zhou Y, Zhao G, Bauer IK, Droit L, Ndao IM, Warner BB, Tarr PI, Wang $D$, Holtz LR. Early life dynamics of the human gut virome and bacterial microbiome in infants. Nat Med. 2015;21(10):1228-34.

27. Lv Z, Peng G, Liu W, Xu H, Su J. Berberine blocks the relapse of Clostridium difficile infection in C57BL/6 mice after standard vancomycin treatment. Antimicrob Agents Chemother. 2015;59(7):3726-35.

28. Hsiao A, Ahmed AM, Subramanian S, Griffin NW, Drewry LL, Petri WA Jr, Haque R, Ahmed T, Gordon Jl. Members of the human gut microbiota involved in recovery from Vibrio cholerae infection. Nature. 2014;515(7527): 423-6.

29. Langille MG, Zaneveld J, Caporaso JG, McDonald D, Knights D, Reyes JA, Clemente JC, Burkepile DE, Vega Thurber RL, Knight R, et al. Predictive functional profiling of microbial communities using $16 \mathrm{~S}$ rRNA marker gene sequences. Nat Biotechnol. 2013;31(9):814-21.

30. Arboleya S, Sanchez B, Solis G, Fernandez N, Suarez M, Hernandez-Barranco AM, Milani C, Margolles A, de Los Reyes-Gavilan CG, Ventura M et al: Impact of Prematurity and Perinatal Antibiotics on the Developing Intestinal Microbiota: A Functional Inference Study. Int J Mol Sci 2016, 17(5).

31. Leung MH, Chan KC, Lee PK. Skin fungal community and its correlation with bacterial community of urban Chinese individuals. Microbiome. 2016;4(1):46.

32. Liu L, Zhao H, Zhang Y, Wang J, Che Y, Dong C, Zhang X, Na R, Shi H, Jiang $L$, et al. Neonatal rhesus monkey is a potential animal model for studying pathogenesis of EV71 infection. Virology. 2011;412(1):91-100.

33. Marx PA, Maul DH, Osborn KG, Lerche NW, Moody P, Lowenstine LJ, Henrickson RV, Arthur LO, Gilden RV, Gravell M, et al. Simian AIDS: isolation of a type $D$ retrovirus and transmission of the disease. Science. 1984; 223(4640):1083-6.

34. Zhang XX, Wu QF, Yan YL, Zhang FL. Inhibitory effects and related molecular mechanisms of total flavonoids in Mosla chinensis maxim against H1N1 influenza virus. Inflamm Res. 2018;67(2):179-89.

35. Majmudar H, Hao M, Sankaranarayanan NV, Zanotti B, Volin MV, Desai UR, Tiwari V. A synthetic glycosaminoglycan mimetic blocks HSV-1 infection in human iris stromal cells. Antivir Res. 2018;161:154-62.
36. Greseth MD, Traktman P. De novo fatty acid biosynthesis contributes significantly to establishment of a bioenergetically favorable environment for vaccinia virus infection. PLoS Pathog. 2014;10(3):e1004021.

37. Lieberherr C, Zhang G, Grafen A, Singethan K, Kendl S, Vogt V, Maier J, Bringmann G, Schneider-Schaulies J. The plant-derived naphthoquinone Droserone inhibits in vitro measles virus infection. Planta Med. 2017;83 (3-04):232-8.

38. Agus A, Planchais J, Sokol H. Gut microbiota regulation of tryptophan metabolism in health and disease. Cell Host Microbe. 2018;23(6):716-24.

39. Stehle T, Peters T, Hartmann L, Schelhaas M: Glycans controlling virus infections: meeting report on the 1st international symposium on Glycovirology Schontal, Germany, 02(-)04. Viruses 2018. May 2018:10(11).

40. Lin LT, Chen TY, Lin SC, Chung CY, Lin TC, Wang GH, Anderson R, Lin CC, Richardson CD. Broad-spectrum antiviral activity of chebulagic acid and punicalagin against viruses that use glycosaminoglycans for entry. BMC Microbiol. 2013;13:187

41. Min BS, Miyashiro $H$, Hattori M. Inhibitory effects of quinones on RNase $H$ activity associated with HIV-1 reverse transcriptase. Phytother Res. 2002; 16(Suppl 1):S57-62.

42. Ohtake S, Arakawa T, Koyama AH. Arginine as a synergistic virucidal agent. Molecules. 2010;15(3):1408-24.

43. Arakawa T, Kita Y, Koyama AH. Synergistic virus inactivation effects of arginine. Biotechnol J. 2009;4(2):174-8.

44. Rabbani MAG, Barik S. 5-Hydroxytryptophan, a major product of tryptophan degradation, is essential for optimal replication of human parainfluenza virus. Virology. 2017:503:46-51.

45. Drewes JL, Croteau JD, Shirk EN, Engle EL, Zink MC, Graham DR. Distinct patterns of tryptophan maintenance in tissues during kynurenine pathway activation in simian immunodeficiency virus-infected macaques. Front Immunol. 2016;7:605.

46. Howe A, Ringus DL, Williams RJ, Choo ZN, Greenwald SM, Owens SM, Coleman ML, Meyer F, Chang EB. Divergent responses of viral and bacterial communities in the gut microbiome to dietary disturbances in mice. ISME J. 2016;10(5):1217-27.

47. Yatsunenko T, Rey FE, Manary MJ, Trehan I, Dominguez-Bello MG, Contreras M, Magris M, Hidalgo G, Baldassano RN, Anokhin AP, et al. Human gut microbiome viewed across age and geography. Nature. 2012;486(7402):222-7.

48. Morgun A, Dzutsev A, Dong X, Greer RL, Sexton DJ, Ravel J, Schuster M, Hsiao W, Matzinger P, Shulzhenko N. Uncovering effects of antibiotics on the host and microbiota using transkingdom gene networks. Gut. 2015;64(11):1732-43.

49. Xiong W, Wang Y, Sun Y, Ma L, Zeng Q, Jiang X, Li A, Zeng Z, Zhang T. Antibiotic-mediated changes in the fecal microbiome of broiler chickens define the incidence of antibiotic resistance genes. Microbiome. 2018;6(1):34.

50. Sharland M, Subgroup SP. The use of antibacterials in children: a report of the specialist advisory committee on antimicrobial resistance (SACAR) Paediatric Subgroup. J Antimicrob Chemother. 2007;60(Suppl 1):i15-26.

51. Lai YC, Chuang YC, Chang CP, Lin YS, Perng GC, Wu HC, Hsieh SL, Yeh TM. Minocycline suppresses dengue virus replication by down-regulation of macrophage migration inhibitory factor-induced autophagy. Antivir Res. 2018;155:28-38.

52. Varghese FS, Kaukinen P, Glasker S, Bespalov M, Hanski L, Wennerberg K, Kummerer BM, Ahola T. Discovery of berberine, abamectin and ivermectin as antivirals against chikungunya and other alphaviruses. Antivir Res. 2016;126:117-24.

53. Zhou N, Pan T, Zhang J, Li Q, Zhang X, Bai C, Huang F, Peng T, Zhang J, Liu $C$, et al. Glycopeptide antibiotics potently inhibit Cathepsin $L$ in the late endosome/lysosome and block the entry of Ebola virus, Middle East respiratory syndrome coronavirus (MERS-CoV), and severe acute respiratory syndrome coronavirus (SARS-CoV). J Biol Chem. 2016;291(17):9218-32.

54. De Burghgraeve T, Kaptein SJ, Ayala-Nunez NV, Mondotte JA, Pastorino B, Printsevskaya SS, de Lamballerie $\mathrm{X}$, Jacobs M, Preobrazhenskaya M, Gamarnik AV, et al. An analogue of the antibiotic teicoplanin prevents flavivirus entry in vitro. PLoS One. 2012;7(5):e37244.

55. Finkbeiner SR, Allred AF, Tarr PI, Klein EJ, Kirkwood CD, Wang D. Metagenomic analysis of human diarrhea: viral detection and discovery. PLoS Pathog. 2008:4(2):e1000011.

56. Morse SS, Mazet JA, Woolhouse M, Parrish CR, Carroll D, Karesh WB, Zambrana-Torrelio C, Lipkin Wl, Daszak P. Prediction and prevention of the next pandemic zoonosis. Lancet. 2012;380(9857):1956-65.

\section{Publisher's Note}

Springer Nature remains neutral with regard to jurisdictional claims in published maps and institutional affiliations. 